\title{
Double aortic arch
}

\author{
R. Nijveldt, T. Germans, A.M. Beek, M.J.W. Götte, A.C. van Rossum
}

A 58-year-old male was referred to our outpatient cardiology clinic for evaluation of atrial fibrillation, fatigue, dizziness and exertional dyspnoea. He had suffered recurrent pneumonia in childhood. Beside an irregular pulse, the physical examination was normal. Electrocardiography showed atrial fibrillation (ventricular response of 60 to 70 beats $/ \mathrm{min}$ ), a right bundle branch block and nonspecific repolarisation abnormalities. Pulmonary vascular redistribution was visible on chest X-ray (figure 1). Transthoracic and transoesophageal echocardiography demonstrated a sinus venosus type atrial septal defect with an anomalous drainage of the right upper pulmonary vein. He was sent for cardiovascular magnetic resonance (CMR) for further evaluation.

CMR demonstrated dilation of the right ventricle, right atrium, caval veins, pulmonary arteries and pulmonary veins and an important systemic to pulmonary shunt. MR angiography demonstrated an abnormal drainage of the right upper pulmonary vein into the superior vena cava, and an anomalous course of the left innominate vein, dorsal to the brachiocephalic trunk. Furthermore, a double aortic arch was found (figure 2), with trachea and oesophagus running through the vascular ring formed by the two arches. There was no tracheobronchial or oesophageal compression. The patient was sent for surgical closure of the atrial septal defect, and showed a substantial clinical improvement one year after correction.

\section{R. Nijveldt}

T. Germans

\section{A.M. Beek}

M.J.W. Götte

A.C. van Rossum

Department of Cardiology, VU University Medical Centre, Amsterdam, the Netherlands

\section{Correspondence to: R. Nijveldt}

Department of Cardiology, Room 6D120, VU University Medical Centre, PO Box 7057, 1007 MB Amsterdam, the Netherlands

E-mail: r.nijveldt@vumc.nl

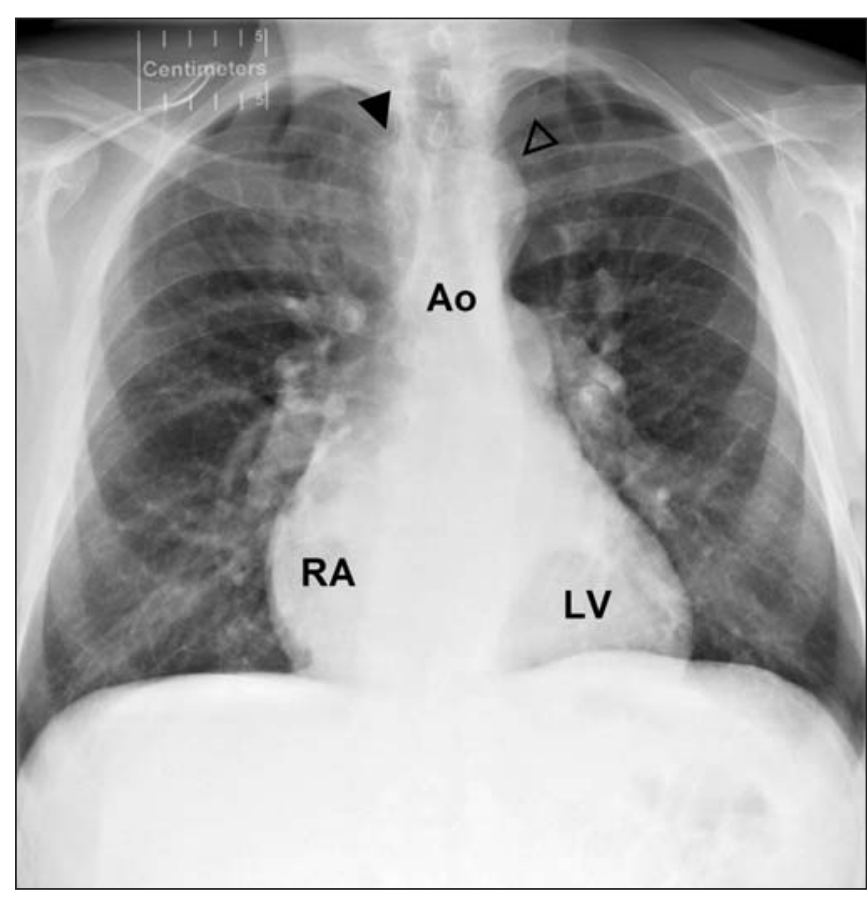

Figure 1. Chest X-ray. Besides pulmonary vascular redistribution, the double aortic arch is visible. The right aortic arch appears as a bright silbouette (dark arrow head) next to a clearer silbouette of the left aortic arch (open arrow head). Ao=aorta, $R A=$ right atrium, $L V=$ left ventricle.

Congenital anomalies of the aortic arch represent less than $1 \%$ of all congenital cardiac defects, and are not known to be associated with other abnormalities. ${ }^{1}$ An anomalous aortic arch results from aberrant development of the embryonic pharyngeal arch system. Common symptoms of a double aortic arch with vascular ring are stridor, respiratory distress, recurrent pneumonia, dysphagia or difficulties with feed, due to tracheobronchial and/or oesophageal compression. Symptoms of airway obstruction predominate in infancy, whereas oesophageal symptoms are more apparent in older children and adults. In this patient, the recurring pneumonia in childhood may have been caused by the atrial septal defect as well as the double 


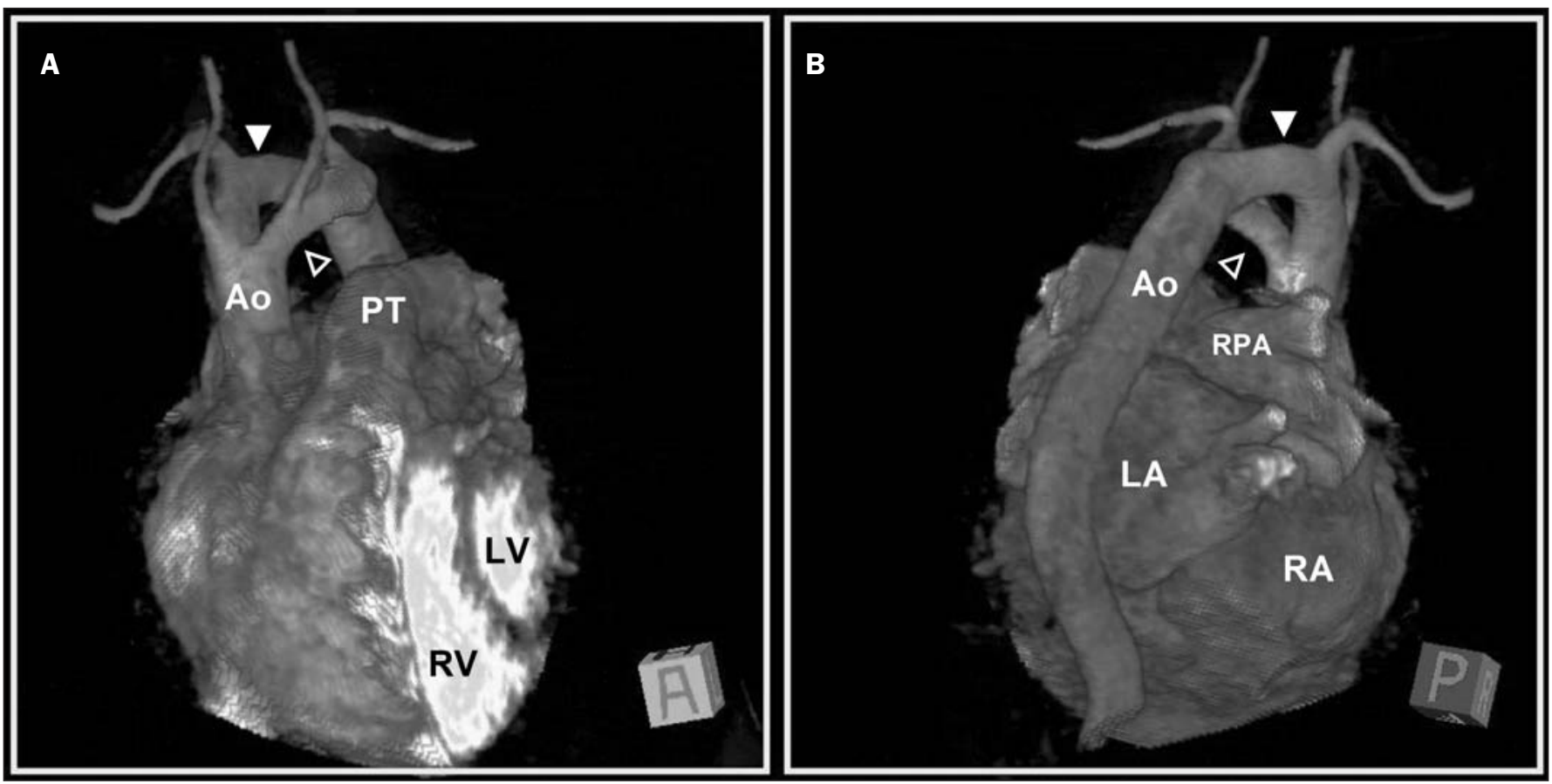

Figure 2. Anterior view (panel A) and posterior view (panel B) of a volume-rendered MR angiogram, demonstrating the main aorta (Ao), which after the ascending aorta splits into a left (open arrow) and right (white arrow) aortic arch, before becoming one descending aorta. $P T=$ pulmonary trunk, $R V=$ right ventricle, $L V=$ left ventricle, $R P A=$ right pulmonary artery, $R A=$ right atrium, LA=left atrium.

aortic arch, whereas the current symptoms were most probably associated with the large systemic to pulmonary shunt and concomitant atrial fibrillation. The co-existence of both an atrial septal defect with aberrant upper pulmonary vein and a double aortic arch is extremely rare.

Evaluation of congenital cardiac malformations often requires a multimodality approach that includes two- and three-dimensional echocardiography, angiography and haemodynamic evaluations, computed tomography, and CMR. In this particular case, CMR provides the optimal single imaging study to noninvasively explore the diagnosis and characterisation of vascular pathology, and evaluate its clinical importance, without the use of ionising radiation.

Moving images illustrating this condition are shown on the journal's website at www.cardiologie.nl.

\section{References}

1 Goldmuntz E. The epidemiology and genetics of congenital heart disease. Clin Perinatol 200;28:1-10.

In this section a remarkable 'image' is presented and a short comment is given.

We invite you to send in images (in triplicate) with a short comment (one page at the most) to Bohn Stafleu van Loghum, PO Box 246, 3990 GA Houten, e-mail: l.jagers@bsl.nl.

'Moving images' are also welcomed and (after acceptance) will be published as a Web Site Feature and shown on our website: www.cardiologie.nl

This section is edited by M.J.M. Cramer and J.J. Bax. 